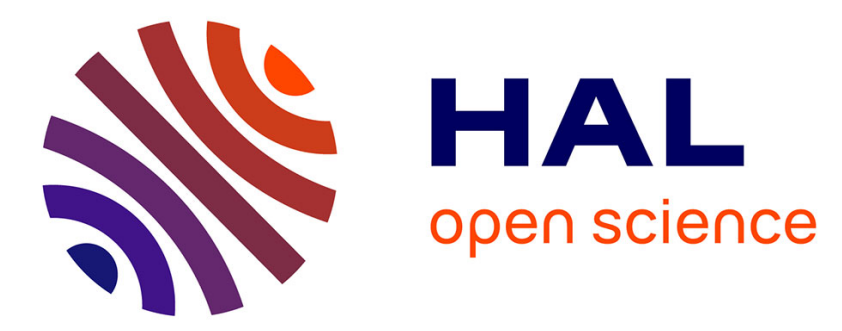

\title{
Vehicle localization in urban canyons using geo-referenced data and few GNSS satellites.
}

Clément Fouque, Philippe Bonnifait

\section{To cite this version:}

Clément Fouque, Philippe Bonnifait. Vehicle localization in urban canyons using geo-referenced data and few GNSS satellites.. IFAC Symposium on Intelligent Autonomous Vehicle, 2007, Toulouse, France. pp.84. hal-00445268

\section{HAL Id: hal-00445268 https://hal.science/hal-00445268}

Submitted on 8 Jan 2010

HAL is a multi-disciplinary open access archive for the deposit and dissemination of scientific research documents, whether they are published or not. The documents may come from teaching and research institutions in France or abroad, or from public or private research centers.
L'archive ouverte pluridisciplinaire HAL, est destinée au dépôt et à la diffusion de documents scientifiques de niveau recherche, publiés ou non, émanant des établissements d'enseignement et de recherche français ou étrangers, des laboratoires publics ou privés. 


\title{
VEHICLE LOCALIZATION IN URBAN \\ CANYONS USING GEO-REFERENCED DATA AND FEW GNSS SATELLITES
}

\author{
Clément Fouque* Philippe Bonnifait* \\ * Heudiasyc UMR CNRS 6599, Université de Technologie \\ de Compiègne, France
}

\begin{abstract}
Stand-alone Global Navigation Satellites System (GNSS) can provide a very accurate real-time positioning in good visibilty and signals conditions. Unfortunately, for a vehicle evolving in urban areas, those conditions are rarely satisfied, leading to debased computed location or lack of solution if less than four SVs are visible. A way to tackle such a problem can consist in fusing the raw measurement from the GNSS reciever with others data sources like proprioceptives sensors. In this paper, the use for computation fix of a priori charted road network data managed by a Geographical Information System (GIS) is presented. Georeferenced data are modeled as segments that can be used as constraints or fused with the pseudo-ranges. Using the residuals, a new method for treating the underlying problem of road selection is proposed. Experimental results illustrate the interesting performance of this approach since the map-matched location can be computed using only 3 satellites and a usual 2D map in urban canyons.
\end{abstract}

Keywords: Outdoor Localization, Global Navigation Satellites Systems, GIS data, tightly coupled data fusion

\section{INTRODUCTION}

The positioning of an intelligent vehicle with respect to a given map is an important issue for many robotics applications. For instance, the map information is very useful for trajectory planning (Remazeilles et al., 2004) or for contextual information retrieval. In some applications, this information can be natural landmarks stored as Geographical Information and used for a precise positioning (Jabbour et al., 2006). Global Navigation Satellites Systems (GNSS) like GPS, Glonass or Galileo are very promising and affordable technologies for robotics.

The problem that consists in localizing a vehicle with respect to a map is known as map-matching. Usually this problem is tackled using GNSS fixes provided by a reciever. This approach has the main drawback to need at least four satellites in line of sight. This condition is rarely satisfied in urban canyon (Georgiev and Allen, 2004). An alternative consists in using a tightly coupled approach in which the map information is used in the computation of the fix. This is the approach considered in this paper. We focus here on the use of a road map provided by cartographers like NavTeQ or TeleAtlas. The available information describes the centerline of the carriageways in a $2 \mathrm{D}$ representation. The main difficulty consists in using such information in the GNSS computation. An approach is proposed in the following to reach this goal. We show how to construct a navigation frame in which the position of the satellites at their emission time is known. By supposing first that the road is known, we show how to compute a location. Then, we propose a strategy to select the most likely road by using the residuals of 
the computation. Experimental results carried out with our experimental show the performance of the approach: the method is able to work in an urban canyon using only 3 satellites. The paper is organized as follows. Section 2 will remind how a stand-alone GPS fix is computed, then several methods for map-aided GPS positioning will be presented in section 3 . In section 4 a simple road selection algorithm will be presented. To conclude, Section 5 will show some experimental results obtained using presented road selection algorithm and map-aided GPS positioning.

\section{STAND-ALONE GPS POSITIONING}

GNSS positioning is based on the multilateration principle: by measuring the time of flight between a receiver and four SVs (Space Vehicles), it is possible to compute the $3 \mathrm{D}$ position of the receiver in a ECEF (Earth Centered, Earth Fixed) frame using the WGS84 Cartesian system. In this section, we present a method to compute an approximate position of the satellites before carrying out the localization computation, under the hypothesis that the receiver clock is approximately synchronized with the GPS time. This method is not necessary for GNSS stand alone computation. It will be useful for the fusion with the GIS data.

\subsection{SVs estimated positions}

SVs broadcast in real-time ephemerids data that contain Keplerian parameters describing their orbits. Given a GPS time-stamp $t_{e}^{i}$, it is possible to compute an estimate position of the SV at this time index in the $\operatorname{ECEF}\left(t_{e}^{i}\right)$, since $\mathrm{ECEF}$ rotates with the earth. The receiver has to solve the following problems:

(1) What is the emission time $t_{e}^{i}$ of the sequence sent by $\mathrm{SV}^{i}$ ?

(2) What was the position $\mathrm{SV}^{i}$ at time $t_{e}^{i}$ in $\operatorname{ECEF}\left(t_{e}^{i}\right) ?$

(3) What is this position in the current ECEF?

The receiver estimates the time of flight $t_{\text {flight }}^{i}$ of a sequence broadcast a SV, by measuring the shift between the emitted frame and its locally generated replica $(\mathrm{C} / \mathrm{A}$ code):

$$
t_{\text {flight }}^{i}=t_{r}-t_{e}^{i}
$$

Where $t_{r}$ is the reception time and $t_{e}^{i}$ is the emission time in the GPS time reference system. $t_{\text {flight }}^{i}$ is in the order of $70 \mathrm{~ms}$. Unfortunately, there is an internal clock bias in the receiver compared to the GPS reference time. At the reception time, the receiver reads its clock $t_{u}\left(t_{r}\right)$. We have:

$$
t_{r}=t_{u}\left(t_{r}\right)+d t_{u}
$$

The emission time is therefore given by:

$$
t_{e}^{i}=t_{u}\left(t_{r}\right)+d t_{u}-t_{\text {flight }}^{i}
$$

If the internal clock bias of the receiver is kept small, then $t_{e}^{i}$ can be approximated by

$$
t_{e}^{i}=t_{u}\left(t_{r}\right)-t_{\text {flight }, \text { measured }}^{i}
$$

Using $t_{e}^{i}$, it is possible to compute an estimated position $X_{\text {sat }}^{i}=\left(x^{i}\left(t_{e}^{i}\right), y^{i}\left(t_{e}^{i}\right), z^{i}\left(t_{e}^{i}\right)\right)$ of $\mathrm{SV}^{i}$ in the frame $\operatorname{ECEF}\left(t_{e}^{i}\right)$ using the broadcast ephemeris. See (Kaplan, 1996) for details.

Now, we need to express the SV position $X_{\text {sat }}^{i}$ in $\operatorname{ECEF}\left(t_{r}\right)$. If we model the earth rotation by a simple 24 hours periodic rotation around $z$ axis (ECEF coordinates), then we have:

$$
\begin{aligned}
& \alpha_{\text {earth }}^{i}=\omega_{\text {earth }} \cdot t_{\text {flight }, \text { measured }}^{i} \\
& { }^{E C E F\left(t_{r}\right)} X_{\text {sat }}^{i}=\operatorname{Rot}\left(z,-\alpha_{\text {earth }}^{i}\right) \cdot{ }^{E C E F\left(t_{e}^{i}\right)} X_{\text {sat }}^{i}(6)
\end{aligned}
$$

\subsection{Receiver position computation}

To compute the position of the receiver, let consider now the pseudo-range measurement $\rho^{i}$ done by the receiver on $\mathrm{SV}^{i}$ :

$$
\rho^{i}=c \cdot t_{\text {flight }, \text { measured }}^{i}
$$

where $t_{\text {flight,measured }}^{i}$ is the measured time of flight. Like the receiver, $\mathrm{SV}^{i}$ has a clock offset:

$$
t_{e}^{i}=t_{s}\left(t_{r}\right)+d t_{s}^{i}
$$

So, we can rewrite the pseudo-range measurement as:

$$
\rho^{i}=c \cdot\left(t_{r}-t_{e}^{i}\right)+c \cdot\left(d t_{u}-d t_{s}^{i}\right)
$$

Where $c$ is the speed of light in vacuum. Let denote $R^{i}$ the geometrical distance between $\mathrm{SV}^{i}$ and the receiver in $\operatorname{ECEF}\left(t_{r}\right)$ frame:

$$
\begin{gathered}
R^{i}=\sqrt{\left(x+x^{i}\left(t_{e}^{i}\right)\right)^{2}+\left(y+y^{i}\left(t_{e}\right)\right)^{2}+\left(z+z^{i}\left(t_{e}\right)\right)^{2}} \\
R^{i}=c \cdot\left(t_{r}-t_{e}^{i}\right)
\end{gathered}
$$

For simplification, let us assume that $d t_{s}^{i}$ can be precisely known using the ephemerids data, the corrected pseudo-range $\rho_{c}^{i}$ is given using Eq.9:

$$
\rho_{c}^{i}=R^{i}+c \cdot d t_{u}
$$

Using Eq.10 and Eq.12, a relationship between corrected pseudo-range measurement for a SV and both receiver position and his internal clock bias has been build. Assuming $n$ visible SVs, we have a non-linear equation system:

$$
\rho_{c}^{i}=h^{i}\left(x, y, z, d t_{u}\right), \forall i=1, \cdots, n
$$


If $n>4$, then the system is also redundant. It is static positioning that can be solved using an iterative least square method (called Newton-Raphson in the following) or Bancroft non-iterative method. Those methods are not explained here. We invite readers to refer to (Kaplan, 1996) and (Yang and Chen, 2001).

\section{USING A ROAD SEGMENT IN THE POSITIONING COMPUTATION}

We now intend to introduce geographical information in the position computation. To illustrate this concept, let us use a digital road map. Under the hypothesis that the current evolution segment is known, we present in this section two ways of including cartographic data.

\subsection{Road maps}

A road map is a database that contains a vectorial description of the road network. Roads are described in a discrete way by their center-line. Data associated with a road are classified in three groups:

- Geographical information: A segment set describing the geometry road

- Topological information: Description of connectivity between road segments

- Semantic information: Road name, speed limit, etc...

Actually, numerical road maps can achieve a metrical precision, which is sufficient to many navigation tasks, like route planning. For memory and real-time constraints, usually the system has in memory a "cache" of the roads around the current position of the vehicle.

\subsection{Working Frame}

In order to compute a valid tightly coupled GNSS/map-matching positioning solution, a common working frame is necessary. Let recall that GPS provide ephemerids data in the WGS84 Cartesian frame whereas maps depict roads making part of the earth surface. So, map nodes and shape points are only described by their longitude and latitude, assuming their elevation equals 0 .

Using the geographical data of the map, let us determine a tridimensional local frame (denoted $l o c)$ such as its plane $(O, i, j)$ be tangential to the WGS84 Earth reference ellipsoid. First, the map points coordinates are converted from the geodetic WGS84 to the Cartesian WGS84 frame. The origin $O$ is chosen to be the origin node of a road close to the estimated position.
The $i$ axis is defined as the first following shape point of the first road if it exists, or the end node if the road is described by a single segment. The $(O, i, j)$ plane is characterized by a geometry point of any other closed road provided that this point doesn't belong to the $i$ axis. Finally, the $k$ axis is chosen such that the local frame be direct.

An homogeneous transform ${ }^{l o c} T_{W G S 84}$ is therefore computed. It contains the rotation and translation terms needed to do the transform. Using ${ }^{l o c} T_{W G S 84}$, the satellite coordinates and the map cache geometry points can be converted in the working frame:

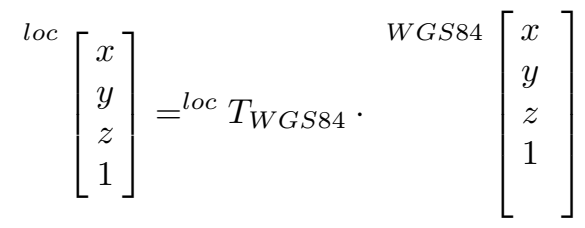

Please notice that the working frame is temporary and valid only for small regions (limited to several kilometers), which tackles the meridian convergence problem.

\subsection{Plane Constraint for Computation}

Let suppose that the good road segment has been selected from the road points given by the GIS. The constraint defined by this selected segment is the piece of a vertical plane (in the working frame), since the elevation of the map is unknown. In practice, we consider the whole plane and we check afterwards that the result matches with the segment.

Taking $A\left(a_{1}, a_{2}\right)$ and $B\left(b_{1}, b_{2}\right)$ as the extremities of the segment, the segment defines a straight line:

$$
y=b_{1}+\frac{b_{2}-a_{2}}{b_{1}-a_{1}} \cdot\left(x-a_{1}\right)
$$

The geometrical equation of this constraint means that only the computation along $(x, y)$ is constrained which can be written by:

$$
y=f_{1}(x)
$$

\subsection{First method: Unknown Elimination}

This method has been proposed by Cui and Ge in (Cui and Ge, 2003). The idea is to eliminate a variable using the constraint equation. Introducing Eq.16 in Eq.10, the geometrical distance between the receiver and $\mathrm{SV}^{i}$ can be rewritten as:

$$
R^{i}=\sqrt{\left(x+x^{i}\left(t_{e}\right)\right)^{2}+\left(f_{1}(x)+y^{i}\left(t_{e}\right)\right)^{2}+\left(z+z^{i}\left(t_{e}\right)\right)^{2}}
$$




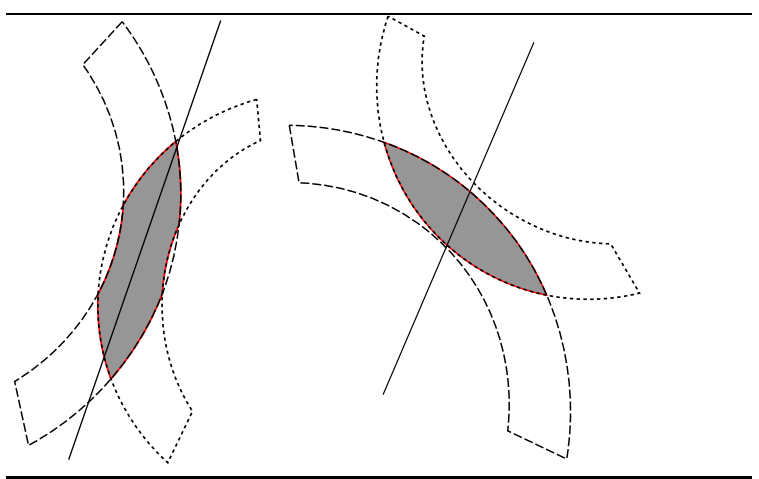

Fig. 1. Incertainity aera according to SV position regarding to the road direction: on the left, 2 satellites are seen transversally; on the right, they are in the direction of the road.

This new expression of the geometrical distance gives a new non-linear system:

$$
\rho_{c}^{i}=g^{i}\left(x, z, d t_{u}\right), \forall i=1, \cdots, n
$$

The problem dimension is now reduced and the minimal number of needed SVs to achieve the computation of positioning solution is 3 . Since the constraint is strong, the computed position belongs to the constraint plane. Please note that its projection onto the map can be outside of the segment.

\subsection{Second method: Plane Fusion}

This methods has been proposed by S. Syed and M.E. Cannon in (Syed and Cannon, 2005). Using the segment parameters, a new observable is built. Therefore, it is possible to add a new equation to the observation model defined using Eq.10:

$$
\begin{gathered}
\left(b_{1}-a_{1}\right) \cdot a_{2}+\left(a_{2}-b_{2}\right) \cdot a_{1}=\left(b_{1}-a_{1}\right) \cdot y+\left(a_{2}-b_{2}\right) \cdot x \\
\rho_{c}^{n+1}=h^{n+1}\left(x, y, z, d t_{u}\right)
\end{gathered}
$$

With this additional measurement and at least three SVs, the positioning solution can be computed. Contrary to the unknown elimination method, the computed solution doesn't belong to the constraint plane defined by the road segment.

\subsection{SVs position relative to road heading}

The geometrical configuration of the SV versus the current segment is crucial. Let consider $2 \mathrm{SVs}$ the positions of which are projected onto the twodimensional map frame thanks to their azimuth and elevation angles. Theirs measurements can be compared to circles of radii $\rho \pm \epsilon$ where $\epsilon$ defines the uncertainty of the measurement. Assuming the vehicle is moving on a charted road, the longitudinal precision provided by the GNSS is more important to achieve a good positioning.
According to Fig.1, one can intuitively notice that if the SVs are located in the direction of the road, they provide a better positioning information than those orthogonal to the road.

\section{ROAD SELECTION ALGORITHM}

We have seen how a road segment information can be introduced into the positioning solution computation. A road selection algorithm is now proposed in order to select the evolution segment that best matches the current GNSS observations.

\subsection{Candidate segments extraction}

For each segment in the road cache, a tightly coupled positioning solution is computed using the unknown elimination method described previously in Sec.3.4 in order to determine the corresponding matched point. Therefore, a non-linear equation system like Eq.18 is solved for each segment. A fix solution is computed using the Newton-Raphson Least Squares iterative solver. A segment can be considered as a candidate if:

- The projection of the fix onto the reference plane $(0, i, j)$ belongs to the segment.

- The fix elevation is close to 0 in the local frame (i.e. lower than a threshold).

Please note that this stage can provide no segment. This can indicate large map errors or a bad GNSS observations.

\subsection{Most likely segment selection}

As the positioning solution is computed using a Newton-Raphson iterative solver with a fixed number of iteration, we suggest to use the residuals $\varepsilon$. Indeed, they allow defining a consistency value in order to choose the most probable segment:

$$
\|\varepsilon\|=|Y-H \cdot d X|
$$

where:

- $Y$ is the measurement

- $H$ is the Jacobian matrix of the observation equation

- $d X$ is the variation of the estimate with respect to the previous one.

Without any a priori information on the vehicle position, the segment with the better consistency is chosen as the most likely segment. Otherwise, if the vehicle position on a segment is known with good accuracy at one moment, then a connex candidate segment will be preferred. 


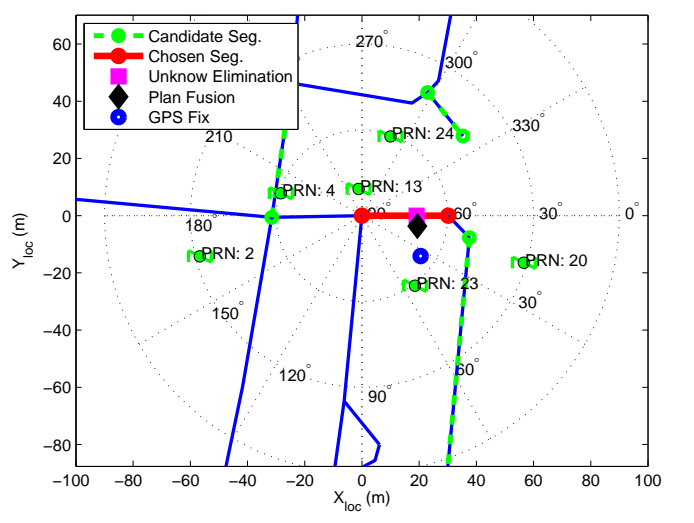

Fig. 2. Road selection results and positioning solution using all visible SVs

\section{FIRST EXPERIMENTAL RESULTS}

\subsection{Methodology}

Experiments have been carried out in two stages. The first stage dealt with data recording and the second with data exploitation. Data has been recorded using our laboratory experimental vehicle strada and a GPS receiver type Trimble 5700 in a stand-alone mode. The data recording has been done on a road next to the lab and this road has been well identified in the geographical database. SVs measurement were recorded using Rinex 2.10 observation file format and the corresponding navigation file has been used.

In this section, we analyse the road selection algorithm and the tightly coupled GNSS-Map fusion using a single fix. For simplicity, the local frame has been set along the current evolution segment. Therefore, the $(O, i)$ axle is coincide with the good segment. Please notice that this segment in reality is not East oriented. Moreover, the SVs used in position computation have been superposed on the map using a skyplot graphic which allows to estimate their elevation and azimuth angles respectively with the origin of the local frame.

\subsection{Road selection}

As shown by Fig.2, let consider what happens if all the 6 visible SVs are used for the computation. Four segments are claimed to be candidate (those plotted in dash) and the current evolution segment has been correctly chosen as the most likely segment (plotted in bold). As presented in section 3 , the positioning solution computed using the unknown elimination method has eliminated the incorrect segments because they have provided solutions outside of the segments or too far from the horizontal frame of the map. Moreover, if we examine the result of the autonomous GNSS fix, we can observe a significant bias in the map data

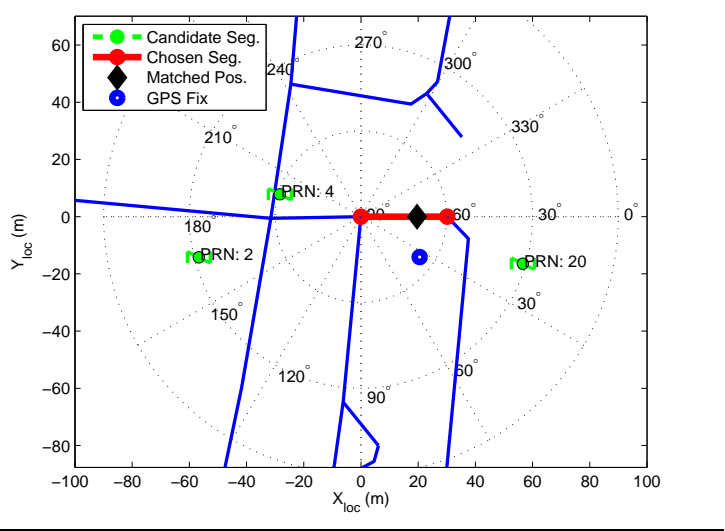

Fig. 3. Road selection and position using three SVs near to the road axle

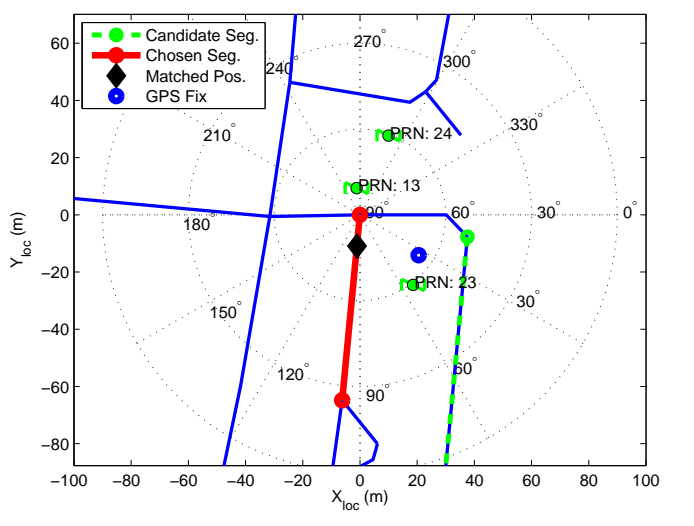

Fig. 4. Road selection and position using three SVs orthogonal to the road axle

( 12 meters). This result show that the presented algorithm is efficient for a simple road selection since there is little ambiguity due to the other candidate segments. The relevance of the proposed tightly coupled GNSS-Map matching using the constraint plane is therefore showed.

\subsection{Impact of SVs configuration}

Let us now appreciate experimentally the impact of SVs configuration with respect to the road in the positioning solution computation. As shown on Fig.3, only the SVs with azimuth near to road heading are used whereas, on Fig.4, the SVs used are orthogonal to the road direction.

We can see that, when the SVs are spread along the segment axle, the correct evolution segment is the only one claimed to be a candidate. When using the SVs which are not along the segment axle, the correct evolution segment doesn't belong to the candidate segments list and so the result of the computation is incorrect. Furthermore, we can notice that all the candidate segments direction are near to the axis made by the SVs and, in this case, orthogonal to the evolution segment. This result proves experimentally the analysis done on 


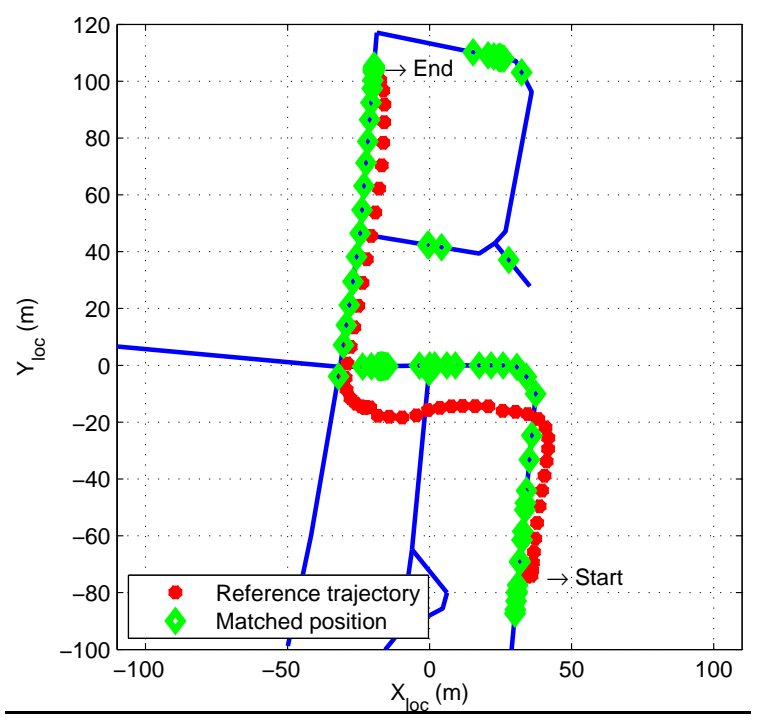

Fig. 5. Map-matched trajectory using the plane fusion method and only $3 \mathrm{SVs}$

Fig.1: in a urban canyon the visible satellites are naturally well configured for a tightly coupled GNSS-Map computation and only $3 \mathrm{SVs}$ can be sufficient.

We have also remarked that, when using few SVs, the positioning solution computed with both methods presented in section 3 are equivalent. This comes from the fact that, when using few SVs, the weight of the constraint plane in the observation model grows up and so, attracts the matched point next to the road segment. With 3 $\mathrm{SVs}$, both methods give the same result.

\subsection{Test with 3 SVs in urban canyons}

By Post-Processing in a Kinematic mode (PPK) the T5700 raw data, a reference trajectory has been built. To test our method, we have eliminated several SVs in the computation in order to create urban canyons with a bad satellites visibility reduced to $3 \mathrm{SVs}$. The positioning solution has been computed using the plane fusion method and the results of the experiment are given on Fig.5. It should be noticed that most of the positioning solutions are well matched despite a significant North offset of the map which induces some missmatches. This first results are very encouraging and show that the proposed positioning method can be efficient in urban areas, even if no tracking of the previous segment has been considered here. Please also note that, at each step, a likely segment has been selected, allowing the computation of a positioning solution.

\section{CONCLUSION AND FUTURE WORK}

In this paper, we have described two methods to fuse road map data with GNSS rough mea- surements (L1 pseudo-ranges). This approach has several advantages. First, as shown by the experiments, it is possible to use only three satellites to compute a fix. Secondly, since the selection of a segment is necessary, the map-matching problem can be solved using the residuals of this computation. The main difficulty arises from the need to compute a GNSS fix using the pseudo-ranges and particularly to locate the satellites thanks to the ephemerid data in a frame attached to the map. We have proposed a method that supposes that the clock drift of the receiver is small. Therefore, the position of the satellites at their emission times can be easily determined in the frame of the map. In order to solve the segment selection problem, we have proposed a simple search strategy. The results that we have obtained are very encouraging since the method is able to retrieve the good segment. We have also confirmed experimentally that the satellites that are the most interesting for the solution computation are those that are in the axle of the road, which is the situation occurring in urban canyons. The perspective of this research is on the use of a dynamic state observer (for instance a Kalman filter) to take benefice of the road connectedness, particularly while approaching junctions. Moreover, we plan also to use WAAS/EGNOS corrections to increase the reliability of the method.

\section{REFERENCES}

Cui, Y.J. and S.S. Ge (2003). Autonomous vehicle positioning with gps in urban canyon environments. IEEE Trans. on Rob. and Aut. 19, 15-25.

Georgiev, A. and P. K. Allen (2004). Localization methods for a mobile robot in urban environments. IEEE Trans. on Rob. and Aut. 20, 851-864.

Jabbour, M., Ph. Bonnifait and V. Cherfaoui (2006). Management of landmarks in a gis for an enhanced localisation in urban areas. In: IV2006 IEEE In. Veh. Symp.. Tokyo, Japan.

Kaplan, Elliot D. (1996). Understanding GPS: principles and applications. Artech House.

Remazeilles, A., F. Chaumette and P. Gros (2004). Robot motion control from a visual memory. IEEE Int. Conf. on Rob. and Aut., ICRA'O4 4, 4695-4700.

Syed, S. and M.E. Cannon (2005). Map-aided gps navigation. GPS World.

Yang, M. and K-H. Chen (2001). Performance assessment of a non-iterative aglorithm for global positioning system (gps) absolute positioning. Proc. Natl. Sci. Counc. ROC (A) 25, 102-106. 